\title{
AutoCAD Add-on for Simplified Design of Microfluidic Devices
}

\author{
Wallace KUNIN, Navid KESHAVARZIAN, and Bin WANG* \\ Department of Chemistry, Marshall University, Huntington, WV 25755 \\ *e-mail:wangb@marshall.edu
}

(Received: March 1, 2010; Accepted for publication: June 4, 2010; Advance publication: July 31, 2010)

\begin{abstract}
The development of polydimethylsiloxane-based microfluidic devices with integrated controlling components has led to the translation of many bench-top biological processes to microdevice platforms. While many biomedical scientists recognize the applicability of microdevices to their research, their lack of engineering background and training in computer-aided design (CAD) makes them unable to design the desired microchips using AutoCAD software, which is the universal first step for chip fabrication. In this work, we used the .NET platform to develop a user-friendly AutoCAD add-on feature that includes the ability to create channels, loops, valves, and punches, as well as channel to loop connections, valve to channel connections, valve to loop connections, channel to channel connections, and punch to channel connections. This add-on feature greatly simplifies the process of AutoCAD drawing, which allows people not familiar with AutoCAD to easily learn to use the add-on. For testing purposes, we used the add-on to draw a series of two-layer microchips designed to synthesize luciferase using a coupled transcription-translation system with different capacities. The AutoCAD files for the microfluidic designs were then sent to the Stanford Microfluidics Foundry for chip fabrication. Our results show that the quantity of luciferase produced on the chip is proportional to the capacity of the reaction loop.
\end{abstract}

Keywords: AutoCAD add-on, Microfluidic device, Polydimethylsiloxane

\section{Introduction}

Microfluidic systems that manipulate biological and/or chemical processes at the microliter or nanoliter scale were developed in the 1990s $[1,5,6]$. Compared to conventional bench-top setups, microfluidic devices have many advantages, including dramatically reduced consumption of reagents and analytes, reduced time of analysis, and enhanced reaction kinetics and heat transfer. Traditional microdevices are fabricated using silicon and glass $[1,6]$. In the past decade, polymer materials, especially the elastomer polydimethylsiloxane (PDMS), have become a promising alternative due to the balance between simplicity and performance: 1) PDMS microchips can be easily fabricated without the need for expensive equipment or even a cleanroom; 2) PDMS is compatible with most biological reagents, and provides a robust performance independent of fluid properties; and 3) PDMS is permeable to gas $\left(\mathrm{O}_{2}\right.$ and
$\mathrm{CO}_{2}$ ), and is thus suitable for bacteria and cell culture-related biological processes $[10,14,20]$.

In 2000, Quake's research group used multilayer soft lithography to bond together two or three layers of patterned PDMS [13,17]. The low Young's modulus $(\sim 750 \mathrm{kPa})$ of this soft polymer allows the deflection of one layer (control channel) of elastomer into the other (flow channel) when a small actuation force is applied, thus forming an active monolithic valve. When three valves are actuated on one channel by a binary pattern such as $001,011,010,110,100,101$, where 0 indicates "valve open" and 1 indicates "valve closed", a peristaltic pump results. When two or three valves on a loop are actuated alternately, fluids in the loop are thoroughly mixed. The multilayer PDMS chip design allows the integration of controlling components (valve, pump, mixer, and meter) through a microfluidic platform, making the execution and automation of parallel and/or sequential biological processes 
possible $[11,15]$. Since this development, an increasing number of researchers have implemented this microfluidic approach, and have developed a variety of functioning microdevices for applications such as polymerase chain reaction (PCR), gene ligation, gene transformation, mRNA isolation from single cells, cell culture, cell screening, and protein crystallization $[2-4,7-9,12,18,22]$.

The fabrication of a PDMS microchip can be generally divided into three steps: 1) chip design by two-dimensional Computer-Aided Design (AutoCAD 2D, www.autodesk. com), followed by the printing of a mask based on the design; 2) mold fabrication by soft lithography to transfer the pattern from the mask to a silicon mold; and 3) chip fabrication by micro-molding to transfer the pattern from the silicon mold to a PDMS chip. The second and third steps can be carried out at specialized facilities, such as a university microfluidic foundry (e.g., Stanford University Microfluidics Foundry and Caltech Microfluidic Foundry) or by a chip shop, with both a reasonable price and short turnaround time. However, the foundry or shop does not usually participate in the first step, because chip design is regarded as a research activity rather than a standardized process.

The common problem most biomedical researchers face is the lack of either an engineering background or training in AutoCAD 2D. With the desire to miniaturize and automate time-consuming and labor-intensive bench-top biological processes into a microfluidic chip, bio-scientists often have to find a collaborator in a Biomedical Engineering department or hire a computer scientist/engineer. In order to help bioresearchers with little or no background in AutoCAD to be able to create a microfluidic chip design, we attempted to develop an add-on feature to simplify the drawing process in AutoCAD.

With the ultrafast expansion of technology, creating software that does not quickly become outdated seems to be an oxymoron. However, this is exactly what a program designer must strive for; and therefore, we chose the .NET platform $[16,19,21]$. The .NET platform uses a managed interface (or virtual machine), which means that any programming language that implements .NET gets converted into the Common Intermediate Language (CIL) before being translated to binary code (or machine code) by the Common Language Runtime (CLR). This allows code written by a programmer in a .NET language to be valid for any operating system. Also, if other programmers contribute to the program in the future, the various parts of the program could be written in different programming languages, as long as each implements the .NET Framework. Currently, C\# (C-Sharp) and Visual Basic (VB) are the programming languages that are most fully built around the .NET platform, even though many other languages also implement it. We selected VB because its syntax is more userfriendly than that of $\mathrm{CH}$.

AutoCAD supports the .NET Framework through the provision of a set of libraries, called the AutoCAD ObjectARX (Object AutoCAD Runtime eXtensions) Managed API (Application Program Interface), which give the .NET programmer access to almost everything AutoCAD can do and almost everything stored within AutoCAD. Furthermore, since Autodesk, Inc., the creators of AutoCAD, are also the designers of the AutoCAD .NET API, there is a cohesiveness with AutoCAD inherent in the libraries. In effect, Autodesk has positioned .NET to be the API of choice heading into the future over older technologies such as VBA (Visual Basic for Applications) and AutoLISP (List Processing Language).

In this work, we used the .NET platform to develop an AutoCAD add-on that includes the most widely used components in microfluidic chip design, such as a channel, loop, valve, punch, channel to loop connection, valve to channel connection, valve to loop connection, channel to channel connection, and punch to channel connection. To test our program, we used our AutoCAD add-on to draw a series of two-layer microchips (including both control and flow layers) with different capacities, and sent the AutoCAD file to the Stanford University Microfluidics Foundry for PDMS chip fabrication. The microdevices were designed to synthesize luciferase protein using the $\mathrm{T}_{\mathrm{N}} \mathrm{T}^{\circledR}$ Quick Coupled Transcription/ Translation System (Promega). The light intensities read using a luminometer show that the quantity of luciferase produced on the chip is proportional to the capacity of the reaction loop.

\section{Program setup}

Much of a programmer's time is spent in error prevention, because there are an infinite number of ways that a user can cause a program to malfunction. Therefore, we require users to start with a template file for each of their AutoCAD designs (see Figure 1); in order to save time, to simplify the user's experience, and to limit the range of possible errors. The template is based on the 4-Inch Mask Template that the Stanford Microfluidics Foundry provides, but it does not limit the user solely to Stanford's design standards. The supplied template file simplifies the look of the 4-Inch Mask Template, adds the capability of designing up to four molds per drawing and includes dynamic blocks (combinations of multiple AutoCAD entities, i.e., lines and circles, into single reusable entities with click and drag parameters) specific to microfluidics. 


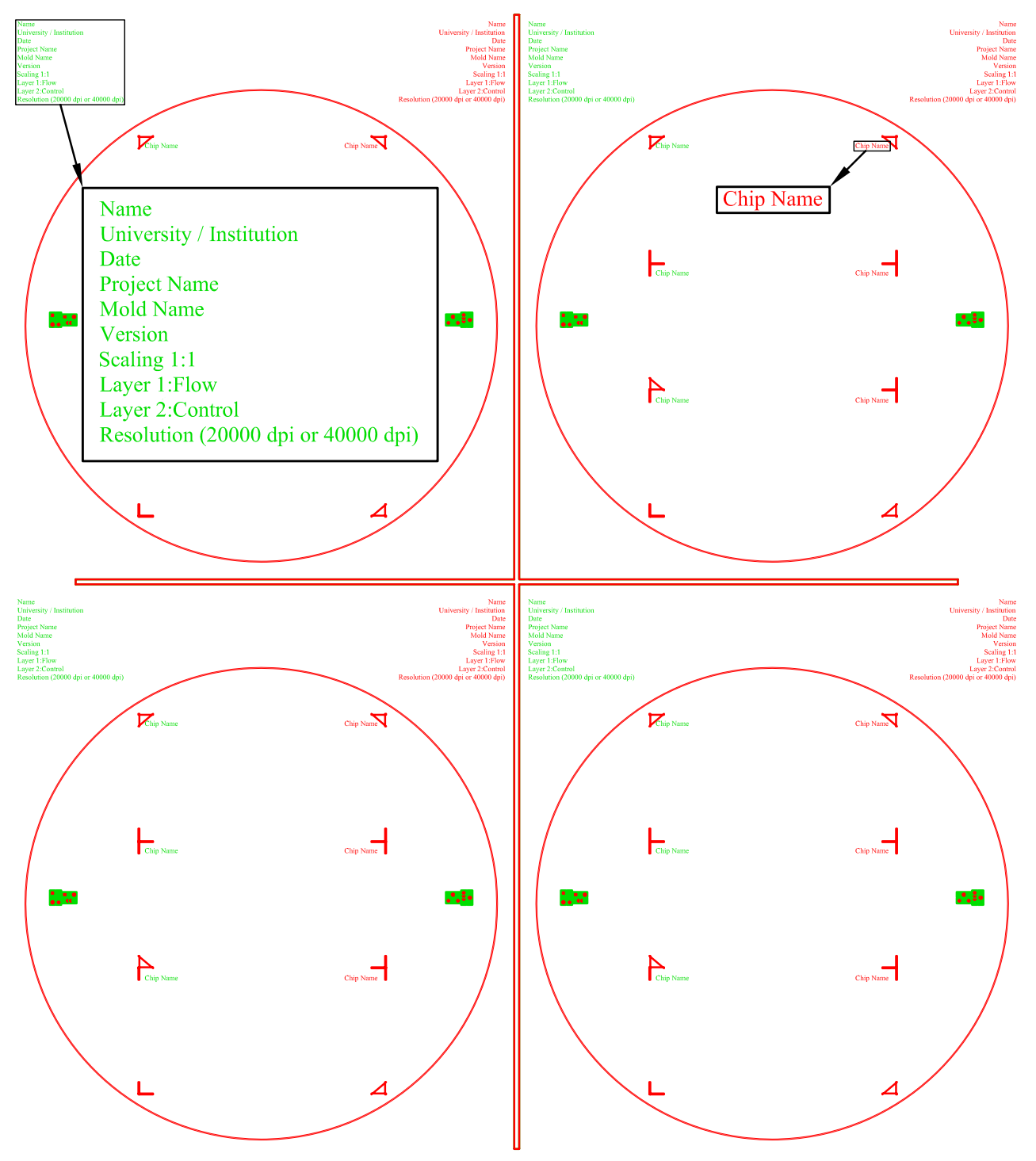

Figure 1. The four-inch diameter mask template, before any drawing has occurred. The text is scaled larger so it can be read more easily.

AutoCAD uses basic drawing entities, such as lines and circles, as the building blocks of drawings. However, it also allows the user to combine certain entities into single entities called blocks. In fact, the database inside each AutoCAD drawing stores the environment the user draws in as a block. In recent editions of AutoCAD, blocks can be made dynamic by adding parameters within the block editor and adding actions to the parameters. This creates grips on the blocks that the user can drag with a computer mouse to adjust the parameters in accordance with the desired actions. These features made dynamic blocks the right candidate to use to represent the basic primitives of the microfluidics design world, such as the valve, punch, alignment mark, and loop.
The only primitive that did not fit the dynamic block mold was the channel. Rather than being a building block itself, the channel's role is better classified as a connector of building blocks. Therefore, channels are built upon a multiline, which is a single entity representing a series of parallel lines.

The AutoCAD UI (user interface), along with the UIs of other programs such as Microsoft Office, used to rely heavily on menus and toolbars. These UIs have evolved into a ribbon (strip of command icons across the top of the screen that is more varied and detailed than a toolbar), and in the case of AutoCAD, palettes (which look like tabs). We chose to use palettes for our UI, and included instructions for how each one is to be used. 
There are four palettes the user can view. The topmost palette is for inserting a channel into the drawing (see Figure 2a). The user selects the desired width of the channel, selects which part of the channel the mouse will lock onto, and then clicks the Draw button. When drawing the channel, the user proceeds as if drawing a line, by clicking where each vertex should be located. Then, the program uses ChannelJig to draw the rest of the channel as offset lines. The second palette from the top is the Punch palette (see Figure 2b). It is simple to use in that it lets the user select one of four punch sizes and then click Draw to insert the desired punch. Third, the Valve palette lets the user select the width of the flow channel that the valve will be on, and adjusts the valve size accordingly (see Figure 2c). Once again, the user simply clicks Draw to begin the process of choosing where to insert the valve into the drawing. Finally, the Flow Loop palette lets the user pick the capacity of the loop, the width of its channel, and the depth of the flow layer on the chip (see Figure 2d). Based on this input, the program calculates the size the loop needs to be. The user then selects which point on the loop the mouse should snap to during the insert operation, and then clicks Draw.

One extremely useful feature that AutoCAD employs within many commands is the concept of dragging. Dragging allows the user to preview the changes being made when moving, stretching, scaling, or rotating an entity or multiple entities. As the user moves the mouse, AutoCAD retrieves either the point where the mouse is located or the angle or distance the point is from a particular base point. This is called sampling. Next, AutoCAD updates the entity or entities in respect to the sampling by moving, stretching, scaling, or rotating. The effect of doing this every time the user moves the mouse is a preview of the changes the user is making before choosing whether to click the mouse to finalize the changes or press Esc to cancel them. The AutoCAD .NET libraries allow the programmer to access dragging through inheriting from two classes, DrawJig and EntityJig. The programmer inherits from the EntityJig class when he or she wishes to drag only one entity; he or she inherits from the DrawJig class when he or she wishes to drag any number of entities.

Another useful AutoCAD window is the DesignCenter. While the DesignCenter contains a variety of features, we used the Blocks section within the Open Documents Tab to allow the user to click and drag any of the microfluidics blocks into the drawing, with their parameters set to defaults (see Figure 3). This provides a second option for the user to edit the parameters. One can use the grips on each dynamic block to edit the parameters after the blocks are already in the drawing.
Because the alignment mark has no parameters to change but simply grips that move it, there was no need to create a tab for it. Instead, it can only be dragged into the drawing from the DesignCenter.

To enable the user to draw a valve, punch, or loop, we created an InsertJig class. Due to the inclusion of dynamic blocks in the template file, the InsertJig simply needs to access the proper block, adjust its parameters to match the options the user selected from the palette, and then position the block according to where the user's mouse is when it is clicked. Each block has a base point that acts as the location that the mouse cursor locks onto as the drag operation occurs. For a punch or valve, the base point is at the center. However, for a loop, the user can choose between the center, and the topmost, bottommost, leftmost, or rightmost point. Finally, InsertJig appends the new block to the database.

One of the most difficult processes for people not familiar with AutoCAD is connecting together entities that are already inserted into the drawing. There is an art to using the various object snaps in AutoCAD that allow connections to be made exactly so that the user does not have to question whether two entities are really touching or whether there is a small indiscernible space between them. To help users with this issue, we have implemented the connect command. When the user types CONNECT, the program prompts the user for two entities. The first entity will move and rotate to connect to the second entity, which remains stationary. From this point, the program offers a set of choices, depending on the entities chosen for the connection. Possible connection types are channel to loop, valve to channel, valve to loop, channel to channel and punch to channel (see Figure 4).

The channel to loop connection is a compilation of three jigs. The first jig allows the user to choose which end of the channel will be connected to the loop. This is done with the CircleJig, which draws a circle around the end of the channel that is closest to the mouse pointer (see Figure 4a). After the user clicks the mouse button, selecting a channel end, the connection proceeds to the AlignRotateJig. This jig determines the point on the loop to which the channel will be fixed. This point can be anywhere on either the inside or the outside circles of the loop. As the user moves the mouse, the jig previews the channel emanating from the point on the loop closest to the mouse pointer at a perpendicular angle (see Figure 4b).

The valve to flow channel connection centers on the concept of move lines. The move lines represent the set of possible points that the valve's center can lock onto along the channel. Once the program determines the lines (see 
(a)

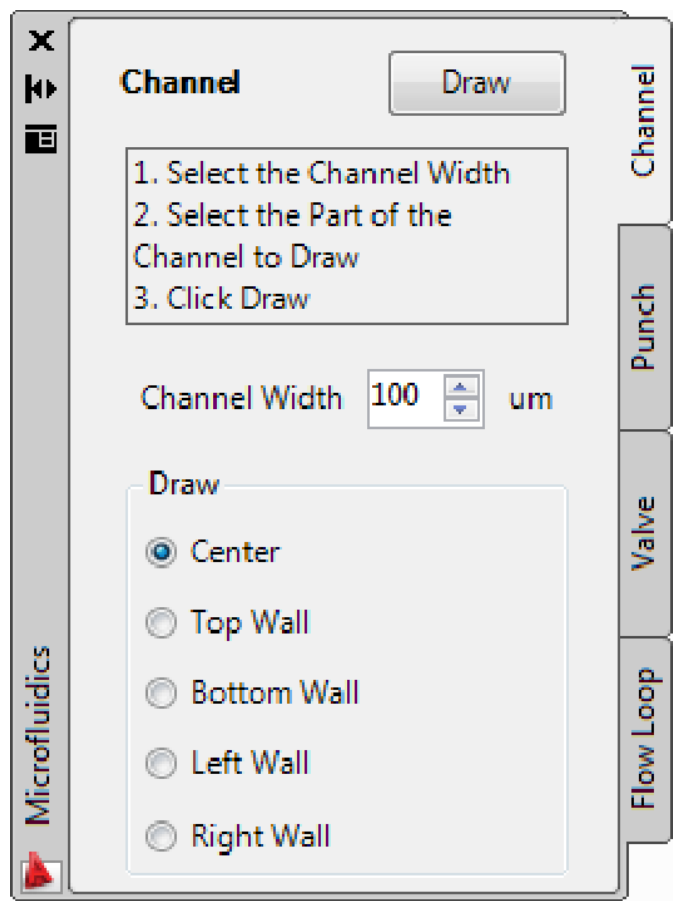

(b)

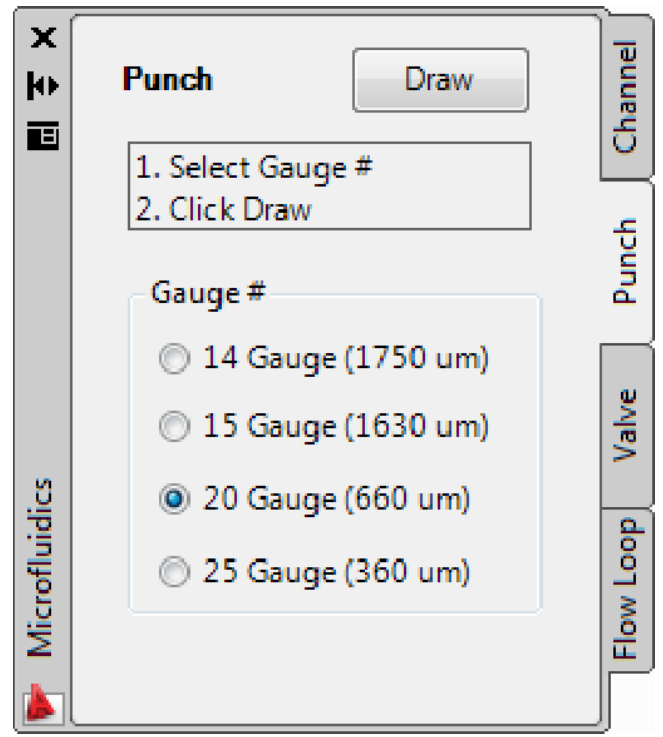

(c)

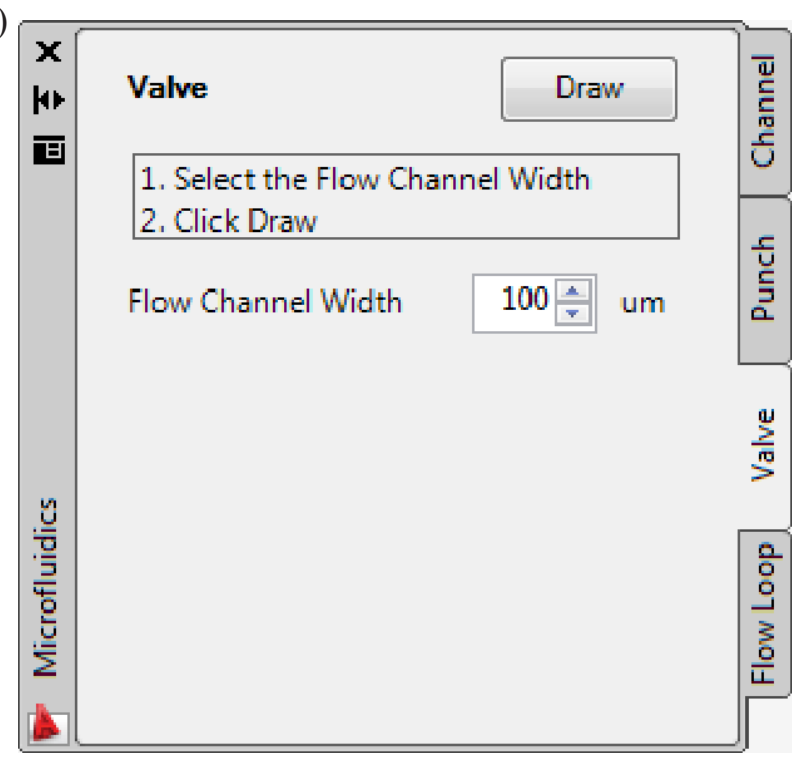

(d) $x$

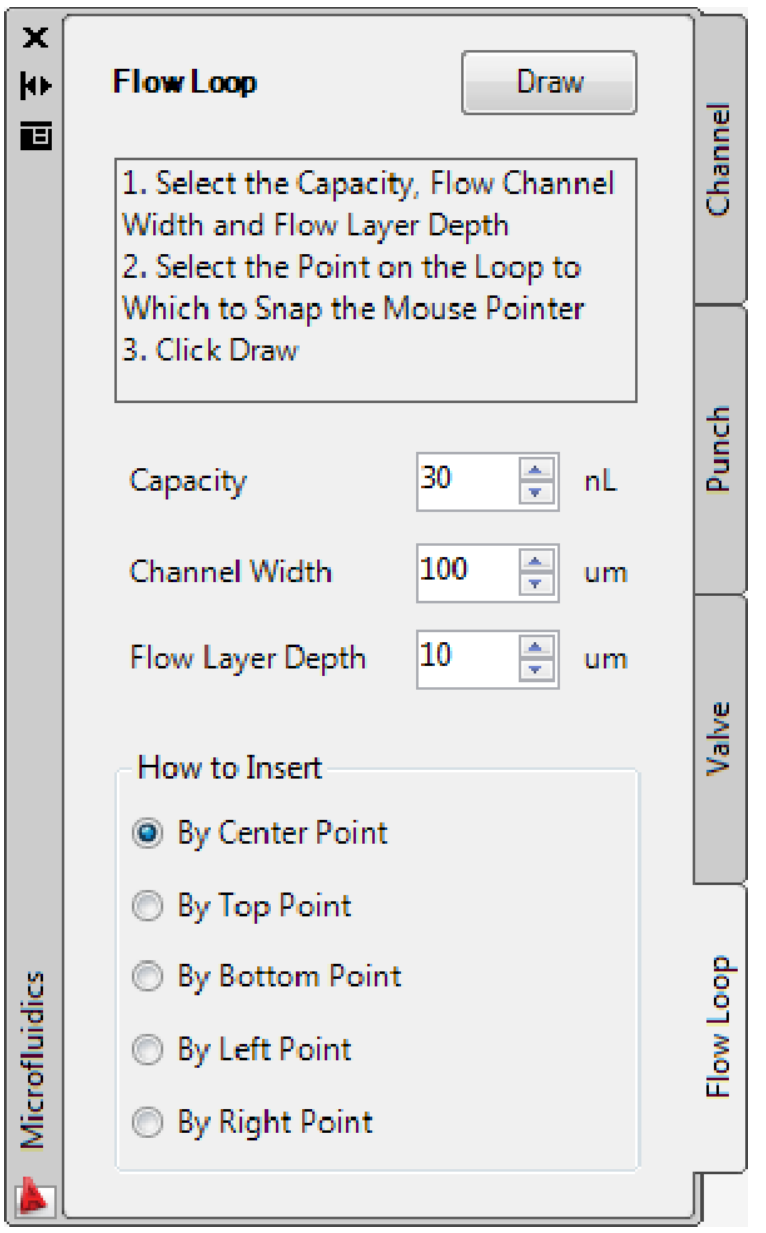

Figure 2. An illustration of our AutoCAD add-on's four tabs and their contents: (a) the channel palette; (b) the punch palette; (c) the valve palette; and (d) the flow layer palette. 


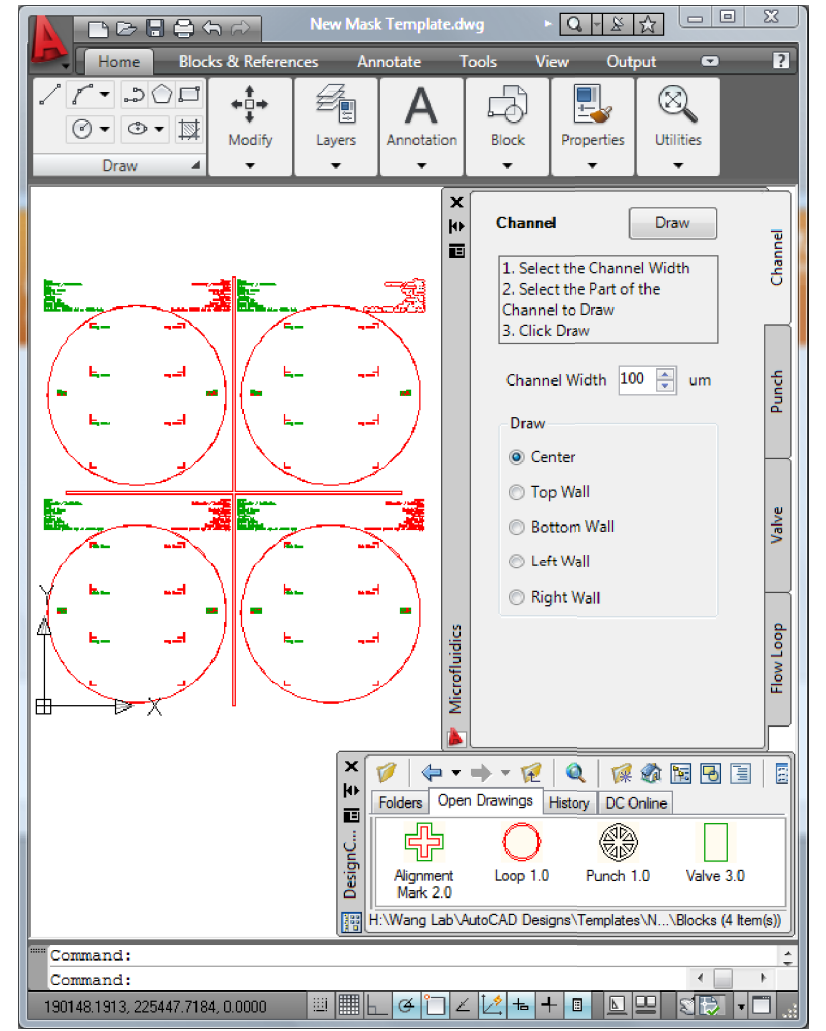

Figure 3. An illustration of the AutoCAD window with the add-on and DesignCenter open.

Figure 4c), they are passed to the MoveJig, which samples the user's mouse location and moves the valve's center to the point on one of the move lines that is closest to the mouse pointer. Once the user clicks, the valve is locked in to its new position.

The valve to loop connection uses the RotateJig to accomplish its task. The jig samples the angle of the user's mouse cursor relative to the center of the loop and moves the valve to the location on the loop at that same angle. Due to the polar tracking setting available in AutoCAD, it is possible to know the exact angle at which each valve is located to the loop (see Figure 4d). This allows easy construction of onloop metering. The user already knows the total capacity of the loop since that is one piece of information entered into the Flow Loop palette when inserting the loop. One also knows the capacity of a reagent needed between two valves if planning to use on-loop metering. With these two pieces of information, the angular distance required between each of the valves can be calculated using the formula:

$\frac{\text { Capacity of Reagent Between Two Valves }(\mu \mathrm{m})}{\text { Total Capacity of the Loop }(\mu \mathrm{m})} \times 360^{\circ}$. (a)

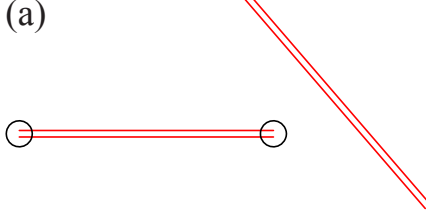

(b)

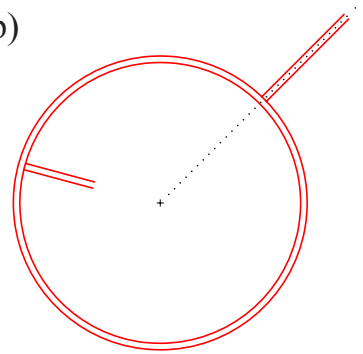

(c)

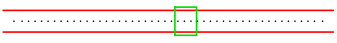

(d)

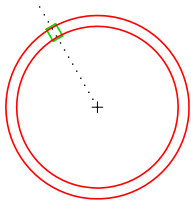

(e)

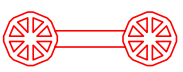

Figure 4. An illustration of the Jigs used for drawing Connections: (a) the CircleJig; (b) the AlignRotateJig; (c) the MoveJig; (d) the RotateJig; and (e) the AlignJig.

The channel to channel connection first makes use of the CircleJig to choose which end of the moving channel will be connected to the stationary channel. The second part of this connection uses AlignJig to move the channel so that its chosen end is aligned with the end of the stationary channel closest to the mouse pointer. Once the user clicks a second time, the moving channel is locked into place (see Figure 4e). The final connection, punch to channel, uses AlignJig in much the same way as the channel to channel connection, substituting the punch for the moving channel.

\section{Description of drawing a chip using the AutoCAD add-on feature}

To test our program, we designed a series of microchips with different capacities $(350 \mathrm{~nL}, 450 \mathrm{~nL}$, and $550 \mathrm{~nL}$ of reaction loop) to synthesize luciferase protein using the $\mathrm{T}_{\mathrm{N}}{ }^{\circledR}$ Quick Coupled Transcription/Translation System (Promega). This kit includes four components for a non-radioactive luciferase control reaction: $\mathrm{T}_{\mathrm{N}} \mathrm{T}^{\circledR}$ quick master mix, methionine $(1 \mathrm{mM})$, T7 luciferase control DNA $(0.5 \mu \mathrm{g} / \mu \mathrm{L})$, and nuclease-free water. The technical manual from Promega indicates that, for a benchtop luciferase positive control reaction with a final volume of $50 \mu \mathrm{L}$, the volume ratio of the four components should be as follows: $\mathrm{T}_{\mathrm{N}} \mathrm{T}^{\circledR}$ quick master mix : $1 \mathrm{mM}$ methionine : $0.5 \mu \mathrm{g} / \mu \mathrm{L}$ T7 luciferase control DNA : nuclease-free water $=40: 1: 2: 7$. Based on this ratio, we calculated the capacities of the reaction chambers needed for the individual components (see Table 1).

Figure 5 illustrates the design of a two-layer PDMS chip 


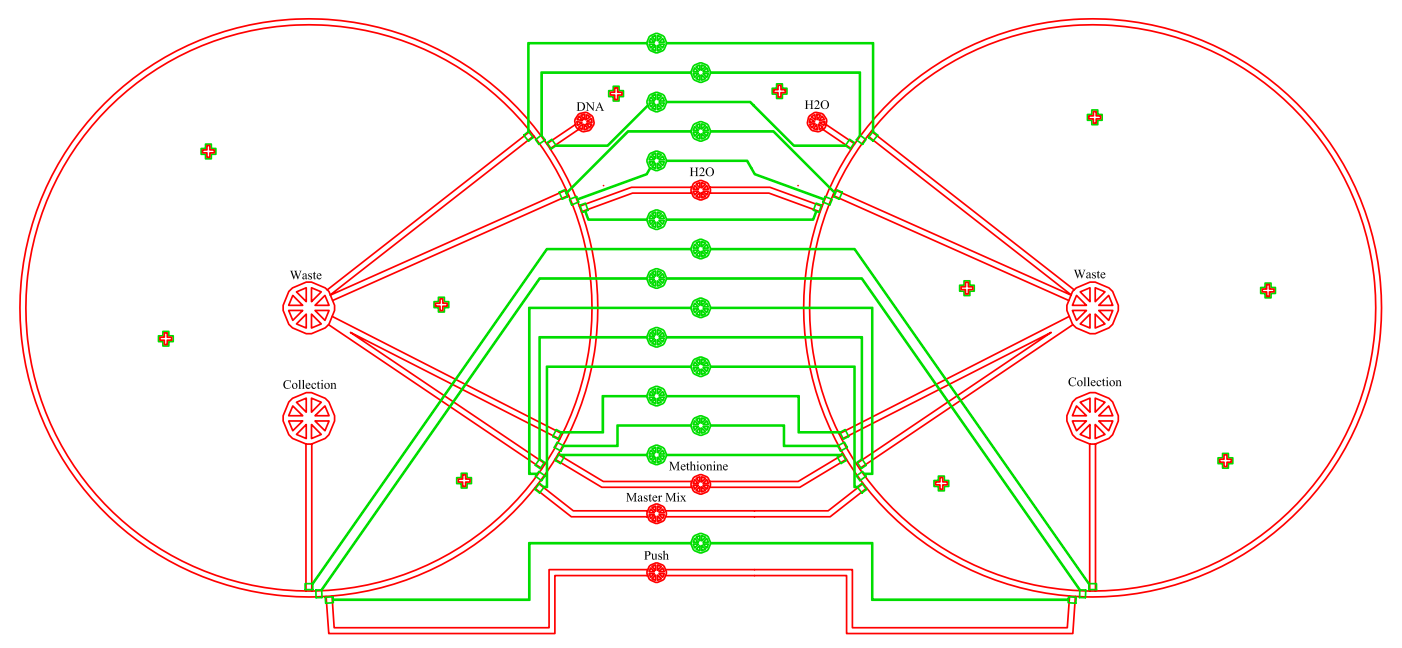

Figure 5. The complete chip design for luciferase protein synthesis. The capacity of each individual loop is $550 \mathrm{~nL}$. The loop on the left-hand side is used for the positive luciferase control reaction; the loop on the right-hand side is used for the negative luciferase control reaction. The labels indicate the function of each punch.

with a capacity of $550 \mathrm{~nL}$ for each reaction loop. Including two separate loops on the chip allows us to run both positive and negative luciferase control reactions simultaneously. The green color represents the control layer, whereas the red color represents the flow layer. The step-by-step description of how to draw the chip design using our add-on feature is provided below.

Step 1: Open AutoCAD. You may automatically be asked which file you wish to open. If so, locate where the New Mask Template.dwg file is and choose it. If you are not automatically prompted, click File > Open from either the big A in the top left corner in AutoCAD 2009 or from the top menu in previous versions of AutoCAD.

Step 2: If the Microfluidics palette set is not visible, type NETLOAD at the command line and locate and select the Microfluidics.dll file. Make sure that endpoint, midpoint, quadrant, center, intersection, and extension object snaps are on by right clicking on the Object Snap icon on the bottom toolbar, then clicking on any of the object snaps that are not yet on and clicking any extra ones that are on to turn them off.

Step 3: Click on the Flow Loop tab. Set the capacity to 550 $\mathrm{nL}$, set the channel width to $200 \mu \mathrm{m}$, and set the flow layer depth to $45 \mu \mathrm{m}$. Click the Draw button. Click somewhere on the left side of one of the chip drawing areas within the file to make sure the entire loop fits within the chip drawing area.

Step 4: Click on the Channel tab. Set the channel width to $200 \mu \mathrm{m}$. Make sure the active layer in AutoCAD is set to Flow (red color). Choose to draw the channel from the center, then click the Draw button. Select the bottom quadrant snap (indicated by a diamond when the mouse is near it) of the inner circle of the loop as the first vertex. Let the polar tracking snap to a 90 degree angle (upward) and then type in 5000 and hit Enter on your keyboard to input this number as the distance to the next vertex. Click Esc to finish.

Table 1. Capacities of chip reaction chambers for luciferase synthesis.

\begin{tabular}{ccccc}
\hline $\begin{array}{c}\text { Capacity of chip } \\
\text { reaction loop }(\mathrm{nL})\end{array}$ & $\begin{array}{c}\text { Capacity of } \mathrm{T}_{\mathrm{N}} \mathrm{T}^{\circledR} \text { quick } \\
\text { master mix }(\mathrm{nL})\end{array}$ & $\begin{array}{c}\text { Capacity of methionine } \\
(\mathrm{nL})\end{array}$ & $\begin{array}{c}\text { Capacity of T7 } \\
\text { luciferase control DNA } \\
(\mathrm{nL})\end{array}$ & $\begin{array}{c}\text { Capacity of nuclease- } \\
\text { free water }(\mathrm{nL})\end{array}$ \\
\hline 350 & 280 & 7 & 14 & 49 \\
450 & 360 & 9 & 18 & 63 \\
550 & 440 & 11 & 22 & 77 \\
\hline
\end{tabular}




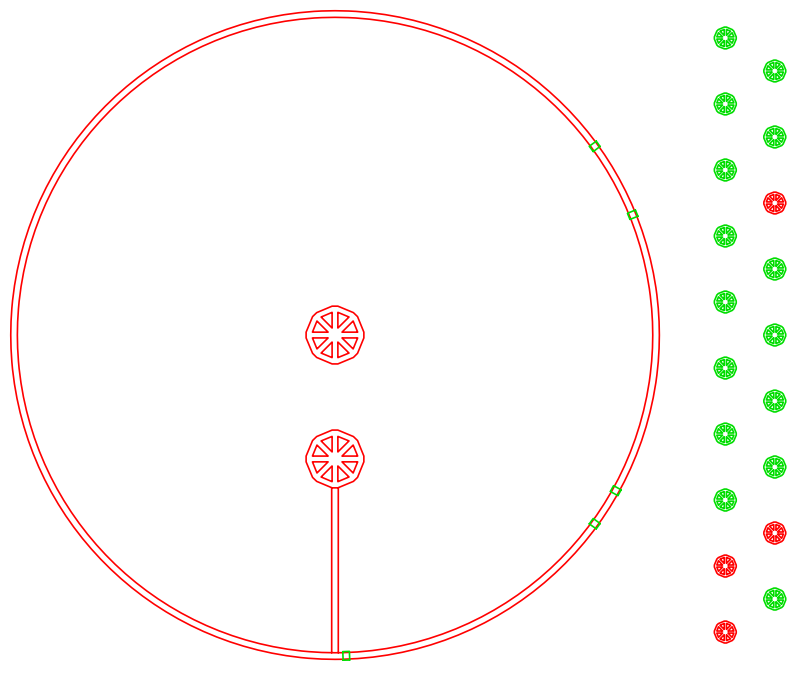

Figure 6. The first stage of chip drawing using the AutoCAD add-on feature. This illustration covers Steps 1-16 in the drawing description.

Step 5: Enter CONNECT at the command line. Select the channel as the moving item and the loop as the stationary object. Choose the bottom end of the channel as the one to connect to the loop. Next, choose to connect the channel once again at the bottom quadrant snap of the inner circle of the loop.

Step 6: Once again, make sure the active layer is set to Flow. Click the Punch tab. Select a 14 Gauge $(1750 \mu \mathrm{m})$ punch and click Draw. Place the punch near the top end of the channel.

Step 7: Enter CONNECT again. Choose the punch as the moving object and the channel as the stationary object. Select the top end of the channel as the align point.

Step 8: Click the Valve tab. Select a $200 \mu \mathrm{m}$ flow channel width. Place the valve near the loop. Repeat this action five times to insert the five valves that will be placed on the loop for this particular chip design.

Step 9: Right click on the polar tracking icon at the bottom of the screen, then click on Settings. Check the box for additional angles. Enter 22, 36, 272, 324, and 331 as values. These values were chosen using the equation at the end of the paragraph on the valve to loop connection with the values from Table 1, then rotating the angles returned so that the valves would all be on the right half of the loop (making each angle $270-360$ or $0-90)$.

Step 10: Use the CONNECT command with the valve as the moving object and the loop as the stationary object. Polar track snap to each of the five angles that you input in Step 9, repeating this command five times to connect each valve to the loop (see Figure 6).

Step 11: Check that the active layer is still set on Flow. Use the Punch tab to place a 14 Gauge punch at the center of the loop using the center object snap.

Step 12: Set the active layer to Control (green color). Insert a 20 Gauge $(660 \mu \mathrm{m})$ punch at the right quadrant snap of the outer circle of the loop. Select the punch and click on the center point. Move the punch right so that the polar tracking snaps to 0 degrees and then type in 2000. Select the punch again, click on the center point again, and move it up until the polar tracking snaps to 90 degrees, then type in 9000 . This moves the punch into the position of the top punch in the left column of punches that are between the two loops in the drawing (see Figure 6).

Step 13: Insert a second 20 Gauge punch at the right quadrant snap of the outer circle of the loop. With the same method as in Step 12, move the punch right $3500 \mu \mathrm{m}$, then up $8000 \mu \mathrm{m}$. This places the punch as the top punch in the right column of punches between the two loops in the drawing (see Figure 6).

Step 14: Select the punch from Step 12, and then type ARRAY to access the ARRAY command. Make sure that Rectangular Array is selected. Set the rows to 10 and the columns to 1 . Next, set the row offset to -2000 and click OK. The column offset is irrelevant.

Step 15: Select the punch from Step 13, and use the ARRAY command. This time, set the rows to 9, leaving the columns at 1 and the row offset at -2000 and click OK. Once again, the column offset is irrelevant.

Step 16: From the two columns of punches just created, click on the bottom two in the left column and the third and eighth from the top in the right column to select them. Set the active layer to Flow to move these punches to the flow layer. They should now be red instead of green (see Figure 6).

Step 17: Insert a valve near the channel at the bottom of the loop. CONNECT the valve (moving) to the channel (stationary) so that the valve is at the very bottom of the channel. Next, move the valve up $50 \mu \mathrm{m}$ using the method described in Step 12 (see Figure 7). 50 is used because the Stanford standard distance between a control layer and a flow layer object that are not forming a valve is $30 \mu \mathrm{m}$. By moving the center of the valve by $50 \mu \mathrm{m}$, the edges are ensured to be at least $30 \mu \mathrm{m}$ away from the loop.

Step 18: Right click on the polar tracking icon, then select Settings again. Delete all the current previously-entered additional angles and then enter 20, 24, 34, 38, 274, 322, 326, 


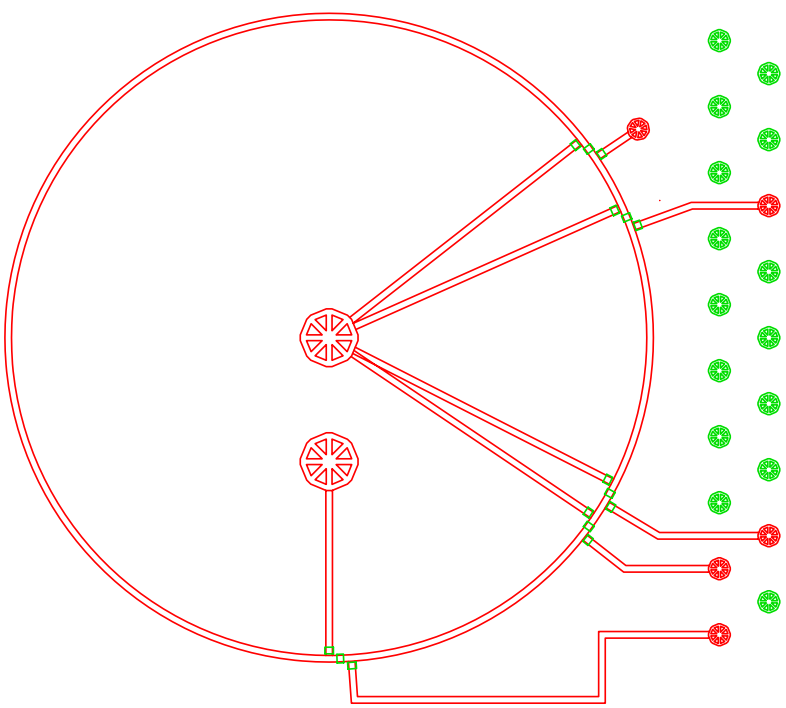

Figure 7. The second stage of chip drawing using the AutoCAD add-on feature. This illustration covers Steps 17-32 in the drawing description.

329 and 333 . We acquired these angles by taking the angles of the valves that we have already placed on the loop and adding and subtracting 2 from each, as the waste and reagent input flow channels are 2 degrees away from each valve, to allow $30 \mu \mathrm{m}$ of room between the valves on the waste and input channels and those on the loop. 270 degrees is not needed as it is already an available polar tracking angle.

Step 19: Set the active layer to Flow and then click the Channel tab. Make sure the channel width is still $200 \mu \mathrm{m}$ and that Center is chosen. Next, click Draw. Set the first vertex of the channel to be the center of the loop. To do this, place the mouse over the loop, then move the mouse to the center of the loop so that the center object snap will appear. Once it appears, click the left mouse button. Set the second vertex to be the intersection with the inner circle of the loop at 24 degrees. Allow the polar tracking to snap to 24 degrees and then look for an $\mathrm{X}$ on the loop, which is the intersection object snap. Use the CONNECT command with the newly-drawn channel as the moving object and the loop as the stationary object. Click near the end of the channel that should be connected to the loop. Next, let AutoCAD polar track snap the mouse to 24 degrees again, and click once more to finish. Do the same to add the channels at 38, 326, and 333 degrees.

Step 20: Type TRIM. Select the outside edge of the punch at the center of the loop and hit Enter on your keyboard. Click on each of the four channels created in Step 19 within the area where they overlap the punch.

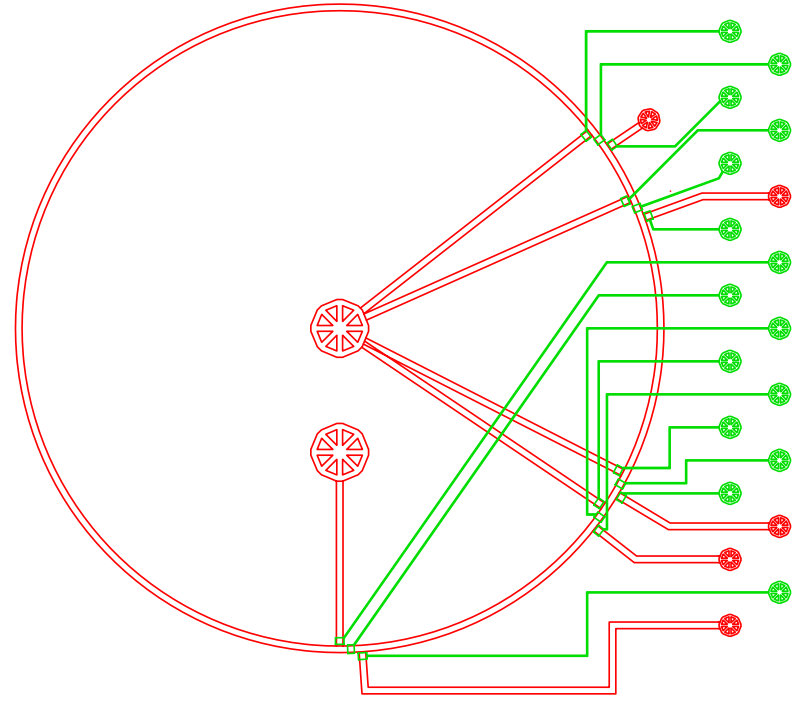

Figure 8 . The third stage of chip drawing using the AutoCAD add-on feature. This illustration covers Steps 33-43 in the drawing description.

Step 21: Delete the four angles from Step 19 from the additional angles in the polar tracking settings. Type 146, 153, 204, and 218 in their place. These are a 180 degree rotation from the four angles in Step 19 so that the valves on these channels can be moved along them in the direction toward the center of the loop.

Step 22: Insert a valve inside the loop. CONNECT it to the top flow channel (of the four created in Step 19), as far to the right as the program will let you. Next, select the valve and click its center point. Let the polar tracking snap to 218 degrees and move it $50 \mu \mathrm{m}$. Do the same for the other three channels from Step 19 using (from top to bottom) 204, 153, and 146 as the angles to snap to.

Step 23: Draw a channel from the center of the loop at 34 degrees that is $11000 \mu \mathrm{m}$ in length. To do so, after selecting the center of the loop as the first vertex, snap to 34 degrees and type in 11000 (see Figure 7).

Step 24: Type TRIM. Select the outer circle of the loop. Click Enter. Click on any part of the channel from Step 23 that is inside the loop. Click Enter again to finish.

Step 25: Insert a 20 Gauge punch near the channel from Step 23. CONNECT it to the end of the channel not connected to the loop.

Step 26: Insert a valve near the channel from Step 23. CONNECT it to the channel as far to the left as possible. Move it $30 \mu \mathrm{m}$ on a 34 degree polar track (see Step 17 for explanation on why $30 \mu \mathrm{m}$ is used). 
Step 27: Draw another $200 \mu \mathrm{m}$ channel choosing the Center radio button. Start at the center object snap of the loop (see Step 19 for explanation). Let AutoCAD snap to 20 degrees. Next, snap to the midpoint of the leftmost line of the top flow punch of the four. Let polar tracking snap to 180 degrees and move the mouse left until this 180 degree polar track intersects with the 20 degree polar track. When AutoCAD snaps to this intersection, click to add a vertex. Click at the midpoint snap used earlier in this step to make it the last vertex. Hit Enter to finish. Draw a second channel, this time choosing the Bottom Wall radio button. Start from the endpoint of the bottom line of the channel just drawn, polar track snap to 0 degrees and move the mouse right until there is an intersection object snap with the punch. Click at the intersection, then hit Enter to finish.

Step 28: Use the TRIM command with the outer circle of the loop as the cutting edge. Then click a point on the channel inside the loop and hit Enter to finish.

Step 29: Insert a valve near the channel from Step 27. CONNECT it to the channel at the leftmost point. Move it $30 \mu \mathrm{m}$ on a 20 degree polar track.

Step 30: Follow Steps 27-29 two more times, substituting 329 and 322 degrees instead of 20 degrees, and using the next two flow punches from the top, respectively.

Step 31: Draw a $200 \mu \mathrm{m}$ flow channel from the center of the loop at 274 degrees with a distance of $11000 \mu \mathrm{m}$, and click to add the second vertex. Add a third vertex at 0 degrees and a distance of $7500 \mu \mathrm{m}$. Next, polar track to 90 degrees. Also, polar track from the midpoint of the leftmost line of the bottom flow punch at 180 degrees until the two polar tracks meet. Click here for a fourth vertex. Add the last vertex at the midpoint snap just used. As before, draw a second channel by its bottom wall from the endpoint of the bottom line of the channel just drawn to the point where a 0 degree polar track makes an intersection object snap with the punch.

Step 32: Follow Steps 28 and 29 for the first channel from Step 31, substituting 274 degrees for 20 degrees (see Figure 7).

Step 33: Set the active layer to Control. Remove all additional angles in the polar tracking settings and set the increment angle to 5. On the Channel tab, set the channel width to $30 \mu \mathrm{m}$ and choose the Center radio button. Use polar tracking, the midpoint snaps of the leftmost line of each punch, and an intersection object snap at each valve to draw the following channels.

Step 34: Start from the topmost punch. Draw a channel from the midpoint snap on the punch (in the same way as before) at 180 degrees until it meets with the 90 degree polar track from the top of the valve that is the leftmost of the top set of three valves, and then continue down to the top of the valve. The channel will not be totally connected. This will be adjusted later. Use the same method with the topmost punch in the right column to the middle valve of the top set of three valves, and the sixth punch from the top in the left column to the top valve in the fourth set of three valves from the top (see Figure 8).

Step 35: Draw a channel from the right valve in the top set of three to the second punch of the left column. Use a distance of $1750 \mu \mathrm{m}$ at 0 degrees from the right point of the valve, followed by a 45 degree polar track to an intersection object snap with the punch.

Step 36: Draw a channel from the midpoint of the top right side of the left valve in the second set of three from the top to the second punch from the top in the right column. Use a 45 degree track, followed by a 0 degree track.

Step 37: Draw a channel from the midpoint of the top right side of the middle valve in the second set of three to the third punch from the top in the left column. Draw at 20 degrees for a distance of $1500 \mu \mathrm{m}$, then use a 60 degree track until it intersects with the punch.

Step 38: Draw a channel from the midpoint of the bottom right side of the right valve in the second set of three from the top to the fourth punch from the top in the left column. Use a 290 degree polar track, followed by a 0 degree track to the punch.

Step 39: Using a 55 degree track followed by a 0 degree track, draw a channel from the top right corner of the left valve in the bottom (fifth from the top) set of three valves to the fourth punch from the top in the right column, and from the middle valve of the same set to the fifth punch from the top in the left column.

Step 40: Draw a channel from the fifth punch from the top in the right column to the midpoint of the top left side of the middle valve in the fourth set from the top. Do this by using a 180 degree track followed by a 270 degree track, and finally a 0 degree track, making sure that no control channels cross each other.

Step 41: Starting from the bottom, draw channels from the bottom punch in the right column to the midpoint of the top right side of the right valve in the bottom set; from the seventh punch from the top in the right column to the right corner of the middle valve of the third set from the top; from the seventh punch from the top in the left column to the right corner of the top valve of the third set from the top; and from the sixth punch from the top in the right column to the right corner of the bottom valve in the fourth set from the top. Use a 180 degree track followed by a 270 degree track, and then another 180 
degree track, making sure not to cross channels nor get them closer than $40 \mu \mathrm{m}$ apart.

Step 42: Draw a channel from the last unconnected punch to the last unconnected valve using a 180 degree track until an intersection object snap.

Step 43: Select the two flow channels going from the loop to the punch at the center of the loop that merge with each other before connecting with the punch. Type in the EXPLODE command. Type in the TRIM command. Hit Enter to select all as cutting edges, and then click the top line of the bottom channel and the bottom line of the top channel to the left of where they intersect (see Figure 8).

Step 44: Select everything drawn so far. Individually deselect all of the punches that are in the two columns at the right, then type in the MIRROR command. Select the midpoint of the top line of any punch in the right column. Next, select the midpoint of the bottom line of any punch in the right column. Hit Enter to say no to erase the source objects.

Step 45: To connect the second punch from the top in the left column to its mirrored channel, start by clicking Draw in the Channel tab. Hold Ctrl and right click the mouse. Select Mid Between 2 Points. Choose the unconnected endpoints of each line of the channel as the two points. Next, use a polar track of 135 degrees, followed by one of 180 degrees. Do the same for the third punch from the top in the left column, substituting 120 degrees for 135 .

Step 46: Click on the unconnected endpoint of each remaining unconnected mirrored channel. Use a 180 degree polar track to snap each channel to the midpoint of the right line of each respective punch. For flow channels, make sure to click on the endpoint of the tiny channel that is at the end and to connect it to the intersection object snap on the punch.

Step 47: Select every control channel without a punch or valve. Enter the EXPLODE command. At each place where a control channel is not connected to a valve or overlaps into the valve, click the endpoint of each line and move it to an intersection object snap with an edge of the valve. Make sure that the closest segment of the channel does not change direction but is rather extended or shortened in the same direction.

Step 48: Type DC to open the DesignCenter. Click the Open Drawings tab. Make sure there is a minus to the left of your file name. Click on Blocks. The Alignment Mark 2.0s can be dropped into the drawing at various places simply through drag and drop operations.

\section{Testing the experimental chips}

The AutoCAD design was sent to the Stanford Univeristy
Microfluidics Foundry for PDMS chip fabrication. Two chips were fabricated for each of the $350 \mathrm{~nL}, 450 \mathrm{~nL}$, and $550 \mathrm{~nL}$ reactions. As shown in Figure 5, the 20 gauge (smaller) punches outside of the reaction loops (in the middle of the design) are used to connect the microchip to the macro world in order to add reactants (in the red flow layer) or to adjust air pressure in order to close a valve (in the green control layer); the 14 gauge (larger) punches at the center and below the center inside the reaction loops receive reaction waste and the luciferase product (see labels in Figure 5). The procedure for operating the chip is briefly described below.

First, apply 10 psi of air pressure to the control layer to close the ten valves on both reaction loops, and the valves on the channels that connect to the Collection Punch and the Push Punch. The valves on the channels that connect to the Reactant Inputs and Waste punches remain open. Second, apply 5 psi of air pressure to push the four reactants $\left(\mathrm{T}_{\mathrm{N}} \mathrm{T}^{\circledR}\right.$ quick master mix, $1 \mathrm{mM}$ methionine, $0.5 \mu \mathrm{g} / \mu \mathrm{L}$ T7 luciferase control DNA, and nuclease-free water) into the loop, where they will fill their individual chambers on the loop and then flow to the Waste Punch. For the negative luciferase control reaction (the loop on the right-hand side in Figure 5), nuclease-free water is used instead of T7 luciferase control DNA. Third, apply 10 psi of air pressure to close the valves on the channels connected to the Waste punches. This step helps the reactants completely fill their individual chambers on the loop, which ensures accurate metering. Fourth, close the valves on the channels connected to the Reactant Inputs. Fifth, open the five valves on each loop in an alternate order, which causes a thoroughly mixing of the reactants within the loop. After mixing, keep all five valves open, and incubate the reaction (i.e., keep the chip) at room temperature for 60-70 min. Sixth, after incubation, open the valves on the channels connected to the Push Punch and the Collection Punches. Use clean, dry air to push the positive and negative control solutions ( $350 \mathrm{~nL}, 450 \mathrm{~nL}$, or $550 \mathrm{~nL}$ ) into their corresponding Collection Punches. Seventh, close the valves connected to the Collection Punches. Add Luciferase Assay Reagent containing beetle luciferin, ATP, and $\mathrm{Mg}^{2+}$ (Promega) into each Collection Punch at a volume of 20 times the reaction volume (e.g., add $11 \mu \mathrm{L}$ of luciferase assay reagent to each Collection Punch for the $550 \mathrm{~nL}$ reaction). Luciferase produced on the chip catalyzes the oxidation of luciferin, thus generating light. For the final step, quickly pipet the mixture from the Collection Punch into a $1.5 \mathrm{~mL}$ nuclease-free Eppendorf tube. Insert the tube into a calibrated GloMax ${ }^{\mathrm{TM}}$ 20/20 Luminometer (Promega), and read and record the light intensity. The raw data are shown in Table 2. 
Table 2. Light intensities read from the luminometer.

\begin{tabular}{ccccccc}
\hline \multirow{2}{*}{ Light intensity } & \multicolumn{2}{c}{$350 \mathrm{~nL}$ reaction loop } & \multicolumn{2}{c}{$450 \mathrm{~nL}$ reaction loop } & \multicolumn{2}{c}{$550 \mathrm{~nL}$ reaction loop } \\
& + reaction & - reaction & + reaction & - reaction & + reaction & - reaction \\
\hline Chip 1 & 2432 & 160 & 3884 & 161 & 5937 & 161 \\
Chip 2 & 2124 & 168 & 4023 & 178 & 5992 & 157 \\
\hline
\end{tabular}

To calculate the average light intensity and standard deviation from each size chip, we first subtracted the light intensity of the negative $(-)$ reaction from that of the positive $(+)$ reaction. The two replicates for each size chip were averaged, and the standard deviation was calculated. Figure 9 shows the trendline for the light intensity versus the capacity of the reaction loop. The error bar represents the standard deviation from the average of two measurements. From the graph, we can see that the light intensity is proportional to the capacity of the reaction loop, which means that the luciferase protein produced on the chip is proportional to the capacity of the reaction loop.

\section{Conclusion and outlook}

The AutoCAD add-on presented in this paper includes the most widely used components in microfluidic chip design, such as channels, loops, valves, punches, channel to loop connections, valve to channel connections, valve to loop connections, channel to channel connections, and punch to channel

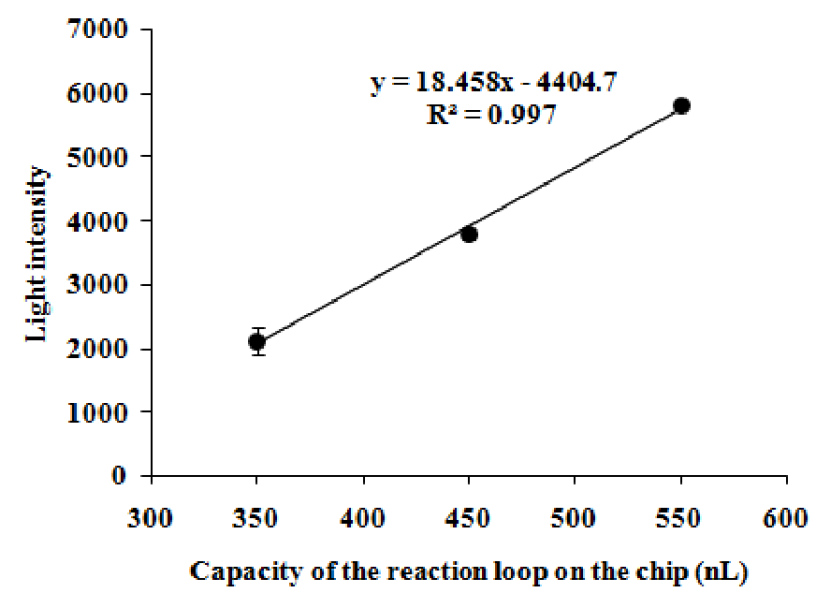

Figure 9. The plot of light intensity produced by the luciferasecatalyzed reaction versus the capacity of the reaction loop on the chip. connections. This user-friendly tool enables investigators (especially biomedical researchers) to draw their desired chip designs, even if they are not familiar with AutoCAD software. The luciferase transcription/translation chip drawn using this add-on performed excellently; we anticipate designing additional microfluidic devices for applications such as RNA structural determination, RNA crystallization, and in vitro translation of proteins.

The AutoCAD add-on was designed with expansion in mind from the start. Dynamic blocks were chosen to be used for many of the base components because they can be combined together into larger blocks and have a great deal of flexibility, since they can be programmed with different parameters. With the current version of the program as a base, expansions planned include the addition of such features as linear and loop metering in their tabs without having to use connections, more basic building blocks (to add to the loop, valve, punch and channel) such as circular and oval storage containers. Also, to be added are compound entities such as storage cells, which are made up of a user-determined number of channels and a multiplexer and demultiplexer containing a combination of valves and channels as an addressing system for the storage cells. In future versions, there will also be additional connections such as loop to channel. Finally, we also have plans to implement totally new features such as control channel routing between punches and valves and channel continuation, allowing a channel to be modified once it is already drawn rather than starting a new channel connected to it. Overall, the goal is to expand the AutoCAD add-on into a more widespread platform to use in both academic laboratories and in the industrial sector meeting the changing needs of the still young microfluidics field.

The authors would like to thank Dr. Michael Norton, Mr. Nathan Crow, and Mr. Jacob Marshall for valuable discussions. This work has been supported by NSF award \# EPS-07-02 Amend 3.1: \#OIA0554328. 


\section{Internet resources}

AutoCAD add-on is available for download at:

http://www.science.marshall.edu/wangb/

\section{References}

[1] C. S. Effenhauser, A. Manz, H. M. Widmer, Anal. Chem., 65, 2637 (1993). doi:10.1021/ac00067a015

[2] R. Gomez-Sjoberg, A. A. Leyrat, D. M. Pirone, C. S. Chen, S. R. Quake, Anal Chem, 79, 8557 (2007). [Medline] doi:10.1021/ac071311w

[3] C. L. Hansen, E. Skordalakes, J. M. Berger, S. R. Quake, Proc Natl Acad Sci U S A, 99, 16531 (2002). [Medline] doi:10.1073/pnas.262485199

[4] C. L. Hansen, M. O. Sommer, S. R. Quake, Proc Natl Acad Sci U S A, 101, 14431 (2004). [Medline] doi:10.1073/ pnas.0405847101

[5] D. J. Harrison, A. Manz, Z. Fan, H. Luedi, H. M. Widmer, Anal. Chem., 64, 1926 (1992). doi:10.1021/ac00041a030

[6] D. J. Harrison, K. Fluri, K. Seiler, Z. Fan, C. S. Effenhauser, A. Manz, Science, 261, 895 (1993). [Medline] doi:10.1126/ science. 261.5123 .895

[7] J W. Hong, V. Studer, G. Hang, W. F. Anderson, S. R. Quake, Nat Biotechnol, 22, 435 (2004). [Medline] doi:10.1038/nbt951

[8] J. Liu, M. Enzelberger, S. Quake, Electrophoresis, 23, 1531 (2002). [Medline] doi:10.1002/15222683(200205)23:10<1531::AID-ELPS1531>3.0.CO;2-D

[9] J. S. Marcus, W. F. Anderson, S. R. Quake, Anal Chem, 78, 3084 (2006). [Medline] doi:10.1021/ac0519460

[10] J. C. McDonald, D. C. Duffy, J. R. Anderson, D. T. Chiu, H. Wu, O. J. Schueller, G. M. Whitesides, Electrophoresis, 21, 27 (2000). [Medline] doi:10.1002/(SICI)15222683(20000101)21:1<27::AID-ELPS27>3.0.CO;2-C
[11] J. Melin, S. R. Quake, Annu Rev Biophys Biomol Struct, 36, 213 (2007). [Medline] doi:10.1146/annurev. biophys.36.040306.132646

[12] E. A. Ottesen, J. W. Hong, S. R. Quake, J. R. Leadbetter, Science, 314, 1464 (2006). [Medline] doi:10.1126/ science. 1131370

[13] S. R. Quake, A. Scherer, Science, 290, 1536 (2000). [Medline] doi:10.1126/science.290.5496.1536

[14] S. K. Sia, G. M. Whitesides, Electrophoresis, 24, 3563 (2003). [Medline] doi:10.1002/elps.200305584

[15] T. Thorsen, S. J. Maerkl, S. R. Quake, Science, 298, 580 (2002). [Medline] doi:10.1126/science.1076996

[16] A. Troelsen, 2008 Pro VB 2008 and the .NET 3.5 Platform (Windows.Net) (Berkeley: Apress)

[17] M. A. Unger, H. P. Chou, T. Thorsen, A. Scherer, S. R. Quake, Science, 288, 113 (2000). [Medline] doi:10.1126/ science.288.5463.113

[18] J. P. Urbanski, W. Thies, C. Rhodes, S. Amarasinghe, T. Thorsen, Lab Chip, 6, 96 (2006). [Medline] doi:10.1039/b510127a

[19] K. Walmsley, 2009 http://through-the-interface.typepad. $\mathrm{com} /$.

[20] B. Wang, Z. Abdulali-Kanji, E. Dodwell, J. H. Horton, R. D. Oleschuk, Electrophoresis, 24, 1442 (2003). [Medline] doi:10.1002/elps.200390186

[21] J. Winters, 2007 VB.NET Programming for AutoCAD Customization (Salt Lake City: VB CAD, Inc.)

[22] J. F. Zhong, Y. Chen, J. S. Marcus, A. Scherer, S. R. Quake, C. R. Taylor, L. P. Weiner, Lab Chip, 8, 68 (2008). [Medline] doi:10.1039/b712116d 
